\title{
Increased Overactive Bladder Symptoms in Primary Sjogren's Syndrome
}

\author{
Figen Tarhan ${ }^{\mathrm{a}, \mathrm{d}}$, Huseyin Tarhan ${ }^{\mathrm{b}}$, Gokhan Keserc, \\ Oguz Mertoglu ${ }^{\mathrm{b}}$, Umit Yildirim ${ }^{\mathrm{b}}$
}

\begin{abstract}
Background: Urinary system involvement is a well-known extraglandular manifestation in primary Sjogren's syndrome (pSS). The term "Overactive Bladder" (OAB) is used to define the symptom complex of urinary urgency, generally accompanied by nocturia, with or without urinary incontinence, in the absence of urinary tract infection or other obvious pathologies. In our study, the frequency of $\mathrm{OAB}$ was investigated in patients with pSS and compared with the frequency observed in normal healthy controls (NHC).
\end{abstract}

Methods: In this cross-sectional study, 50 patients with pSS and $44 \mathrm{NHC}$ were included. For all the participants, the forms of OABValidated 8-question Awareness Tool (OAB-V8) and 3-day voiding diary were completed. The diagnosis of $\mathrm{OAB}$ was made in the presence of OAB-V8 scores higher than 8 . The 3-day means of the parameters in the voiding diary form were calculated and recorded.

Results: The rate of $\mathrm{OAB}$ was found to be $56 \%$ in patients with pSS, while it was $22.7 \%$ in the NHC group. Comparing the pSS patients with the NHC group, significant differences were observed in terms of mean OAB-V8 score $(11.5 \pm 8.8 ; 6.5 \pm 5.3 \mathrm{P}<0.05)$, daytime frequency $(8.4 \pm 3.7 ; 3.6 \pm 2.6 \mathrm{P}<0.05)$ and nocturia $(2.2$ $\pm 1.24 ; 1.2 \pm 1.1 \mathrm{P}<0.05)$. No incontinence was observed in any patients.

Conclusion: $\mathrm{OAB}$ was encountered more frequently in pSS patients than in the NHC group. Since OAB is a treatable symptom complex, it should not be ignored, and symptoms of OAB should

Manuscript accepted for publication May 3, 2013

${ }^{a}$ Katip Celebi University Izmir Ataturk Research and Training Hospital, Department of Internal Medicine, Division of Rheumatology, Izmir, Turkey

${ }^{\mathrm{b}}$ Tepecik Training and Research Hospital, Department of Urology, Izmir, Turkey

${ }^{c}$ Ege University Medical Faculty, Department of Internal Medicine, Division of Rheumatology, Izmir, Bornova, Turkey

${ }^{\mathrm{d}}$ Corresponding author: Figen Tarhan, 126/7 Sok. Hukukcular Sitesi, C Blok, No: 5/3 Evka 3 Bornova, Izmir, Turkey.

Email: eminefigentarhan@hotmail.com

doi: http://dx.doi.org/10.4021/wjnu85w always be searched in pSS patients.

Keywords: Lower urinary tract symptoms; Nocturia; Overactive bladder; Primary Sjogren's syndrome; Voiding diary

\section{Introduction}

Autoimmune rheumatologic diseases may involve different organs and systems. Among these, urinary system involvement is an important and relatively common problem. It has been reported that the severity and the prevalence of lower urinary tract symptoms (LUTS) are increased in Systemic Lupus Erythematosus (SLE), Rheumatoid Arthritis (RA) and Sjogren's syndrome (SS) [1-3].

Primary Sjogren's syndrome (pSS) is a chronic autoimmune rheumatologic disease characterized by lymphocytic infiltration of salivary and lacrimal glands, causing dry eyes and mouth. It can cause extraglandular manifestations such as arthritis, interstitial nephritis, renal tubular acidosis, peripheral neuropathy and palpable purpura, and $90 \%$ of the patients are female [4].

Overactive bladder $(\mathrm{OAB})$ is defined as the symptom complex of urinary urgency, frequency, generally accompanied by nocturia, with or without urgency urinary incontinence, in the absence of urinary tract infection or other obvious pathologies. $\mathrm{OAB}$ has a high prevalence affecting the lives of millions of people worldwide $[5,6]$. Studies have suggested that there could be 17.5 million women in the United States who suffer from this problem [7]. As for Europe, prevalence data has indicated the overall prevalence in women and men over the age of 40 years as $16.6 \%$. The most common symptom reported was daytime frequency (85\%), whereas $54 \%$ complained of urgency and 36\% urgency incontinence. Other symptoms of OAB may be summarized as nocturia, stress incontinence, nocturnal enuresis and often coital incontinence. However, it must be kept in mind that there are various other causes of urgency and frequency [8].

Previous studies have clearly shown that sicca symptoms observed in patients with pSS are mostly the result of autoimmune, autoantibody-mediated autonomic nerve 
Table 1. Demographic Data and Frequency of Overactive Bladder in Primary Sjogren's Syndrome and Normal Healthy Control Groups

\begin{tabular}{lll}
\hline & pSS(n:50) & NHC(n:44) \\
\hline Mean Age (years) & $41.06 \pm 8.70$ & $40.06 \pm 8.46$ \\
Mean Disease Duration (month) & $34 \pm 42.60$ & - \\
Hydroxychioíoquine users & 50 & - \\
Corticosteroid users & 22 & - \\
Methotrexate users & 3 & - \\
Azathiopirine users & 5 & $10(22.7 \%)$ \\
Overactivebladder positive & $28(56 \%)$ & $34(77.3 \%)$ \\
Overactivebladder negative & $22(44 \%)$ & - \\
\hline
\end{tabular}

involvement. This process may also affect the other organs mediated by the autonomic nervous system, including the urinary bladder $[9,10]$.

OAB-Validated 8-question Awareness Tool (OAB-V8) is a screening tool which is used to identify patients suffering from OAB symptoms [11]. OAB-V8 was recently validated for use in primary care settings [12]. Another instrument called 3-day voiding diary, recently reviewed by Cardozo et $\mathrm{al}$, is also widely used to evaluate patients with OAB [7].

In the present study, we aimed to investigate the frequency of OAB in patients with pSS using OAB-V8 and 3-day voiding diary, and tried to find out whether this frequency is different than that observed in normal healthy controls.

\section{Methods}

In this cross-sectional study, 62 female patients with pSS meeting the American-European criteria [13] and being followed up by the Rheumatology Outpatient Department of Katip Celebi University Izmir Ataturk Research and Training Hospital were initially screened. Exclusion criteria were diabetes mellitus, liver disease, congestive heart failure, renal failure, thyroid disease, cerebrovascular disease, uri- nary system stone disease, urogenital anomaly, previous urogenital, abdominal or waist surgery, vaginitis, endometriosis, vaginal prolapse, cystoceles or rectoceles, stress urinary incontinence, suprapubic pain and dysuria, dyspareunia, or sexually transmitted diseases, abnormalities in neurological examination, pathologies determined in complete urine analysis, culture emergence in urine culture antibiogram and malignancies Besides, receiving diuretics, antidepressants or anticholinergics, and the history of pelvic radiotherapy in the past were also exclusion criteria. Based upon these exclusion criteria, 12 patients were not included in this study: positive urine culture antibiogram (n:4); dyspareunia due to vaginal dryness (n:3), stress incontinence (n:3) and painful bladder symptoms (n:2). The remaining 50 patients with $\mathrm{pSS}$ were included in the study. Besides, 44 age matched female healthy controls (NHC) who had no symptoms of autoimmune or connective tissue disorders were also included.

For all the patients with pSS and all the members of the NHC group, OAB-Validated 8-question Awareness Tool forms $(\mathrm{OAB}-\mathrm{V} 8)$ and a 3-day voiding diary were filled in [12]. OAB -V8 questionnaire form included 8 questions on evidence of lower urinary tract symptoms, 2 questions on stress urinary incontinence, one question on incomplete emptying of the bladder during urination. Responses were

Table 2. Mean OAB-V8 Scores for Primary Sjogren's Syndrome (pSS) Patients With or Without Overactive Bladder (OAB) and Normal Healthy Control (NHC) Groups

\begin{tabular}{lll}
\hline & pSS & NHC \\
\hline OAB $(+)$ & $17.85 \pm 6.60$ & $14.1 \pm 5.89$ \\
OAB $(-)$ & $3.45 \pm 2.46$ & $4.29 \pm 2.36$ \\
\hline
\end{tabular}


Table 3. Mean Voiding Diary Results of Patients With Primary Sjogren's Syndrome

\begin{tabular}{llll}
\hline & OAB (-) & OAB (+) & P value \\
\hline Average amount of 24-hour fluid intake $(\mathrm{mL})$ & $2,539 \pm 235.91$ & $2,537 \pm 281.04$ & $>0.05$ \\
Average amount of 24-hour urine output $(\mathrm{mL})$ & $1,998 \pm 233.74$ & $1996 \pm 256.60$ & $>0.05$ \\
Maximal urination volume (mL) & $337 \pm 29.2$ & $169 \pm 32.1$ & $<0.05$ \\
Nocturia & $1.1 \pm 0.40$ & $3.1 \pm 0.85$ & $<0.05$ \\
Daytime Frequency & $4.9 \pm 1.49$ & $9.3 \pm 2.17$ & $<0.05$ \\
\hline
\end{tabular}

OAB: Overactive bladder.

answered on a 6-point Symptom Bother scale ranging from 0 (not at all) to 5 (a very great deal). The 3-day means of the parameters in the voiding diary form were calculated and recorded. By means of voiding diary, daytime frequency of urinating, number of nocturia, amount of 24-hour liquid intake, amount of 24-hour urine output, frequency and severity of incontinence periods, frequency of urgency, and maximum voided urine were determined. The 3-day means of these parameters were calculated and recorded. The patients whose OAB-V8 scores were at least 8 were regarded as having $\mathrm{OAB}$.

This study was approved by the local ethics committee. All the subjects were informed about the study in detail, and written consents were obtained.

Data analyses were carried out on a personal computer using Statistical Package for Social Sciences software (SPSS, Chicago, Illinois) version 16.0, and statistical significance level was set at 0.05 .

\section{Results}

Demographical data of the pSS patients and the NHC group was given in Table 1. Mean age and age range of the pSS and NHC groups were $41.06 \pm 8.70(21-52)$ years and $40.06 \pm$ 8.46 (23 - 51) years, respectively. All the patients with pSS were receiving hydroxychloroquine, 22 patients low dose methyl-prednizolone $(<4 \mathrm{mg} / \mathrm{d})$, and 8 patients diseasemodifying antirheumatic drug (DMARDs) and immunosuppressive drugs including azathiopirine (IS). The frequency of OAB positivity in patients with pSS (56\%) was significantly higher than NHC group $(22.7 \%)(\mathrm{P}<0.05)$, as shown in Table 1. For patients with pSS, mean disease duration was $34.80 \pm 42.60$ months. The OAB frequency did not increase as the duration of the disease was increased $(\mathrm{P}>0.05)$.

The mean OAB-V8 scores of OAB-positive pSS patients $(17.85 \pm 6.60)$ were significantly higher than the NHC group $(14.1 \pm 5.89),(\mathrm{P}<0.05)$ (Table 2$)$. Voiding diary of the patients with pSS was given in Table 3. Both daytime frequency and nocturia rates were significantly higher in $\mathrm{pSS}$ patients having $\mathrm{OAB}$ compared to those without $\mathrm{OAB}(\mathrm{P}<$ $0.05)$.

The distribution of the mean voiding diary parameters and OAB-V8 scores of the patients with pSS and the NHC group were given in Table 4 . When the pSS patients were compared to the NHC group, significant differences were observed in mean OAB-V8 scores $(11.5 \pm 8.8 ; 6.5 \pm 5.3 \mathrm{P}<$ $0.05)$, daytime frequency $(8.4 \pm 3.7 ; 3.6 \pm 2.6 \mathrm{P}<0.05)$ and nocturia $(2.26 \pm 1.24 ; 1.22 \pm 1.1 \mathrm{P}<0.05)$.

No urinary incontinence was observed in any of the patients included in the study.

\section{Discussion}

In the present study we investigated the frequency of $\mathrm{OAB}$ in patients with $\mathrm{pSS}$, and found out that OAB-V8 score, daytime frequency and nocturia were significantly higher compared to NHC group. OAB is a common, problematic and often under diagnosed condition which has a significant negative impact on quality of life. Before discussing the potential mechanisms involved in the occurrence of OAB in $\mathrm{pSS}$, we can have a short look to the pathogenesis of pSS. In pSS, decreased secretion from exocrine glands is partially due to inflammatory destruction of these glands; however parasympathetic dysfunction due to interaction with muscarinic M3 receptors (M3R) on glandular epithelium also contributes to the pathogenetic process. In other words, binding of antimuscarinic autoantibodies to M3R on glandular epithelium further impairs the secretion from the exocrine glands [2].

The irritating bladder symptoms in patients with pSS may be due to detrusor overactivity, caused by involuntary contractions of the detrusor muscle during the filling phase of the micturition cycle, mediated by acetylcholine-induced stimulation of bladder M3R $[10,14]$. However, there remains a paradox to be explained. Since anti-M3R autoantibodies seen in pSS mainly inhibit muscorinic receptors, it seems difficult to reconcile with the symptoms of detrusor overactivity seen in this disease. Interestingly, animal studies suggested that anti-M3R autoantibodies might paradoxi- 
Table 4. Mean Voiding Diary Parameters and OAB-V8 Scores in Patients With Primary Sjogren's Syndrome (pSS) and Normal Healthy Control (NHC) Groups

\begin{tabular}{llll}
\hline & pSS & NHC & P value \\
\hline Mean of OAB-V8 score & & & \\
Average amount of 24-hour fluid intake (mL) & $2,569 \pm 257$ & $2,405 \pm 341$ & $<0.05$ \\
Average amount of 24-hour urine output (mL) & $1,964 \pm 261$ & $1,873 \pm 208$ & $<0.05$ \\
Maximal Urination Volume (mL) & $189 \pm 31$ & $398 \pm 49$ & $<0.05$ \\
Nocturia & $2.2 \pm 1.24$ & $1.2 \pm 1.1$ & $<0.05$ \\
Daytime frequency & $8.4 \pm 3.7$ & $3.6 \pm 2.6$ & $<0.05$ \\
\hline
\end{tabular}

cally inhibit bladder contractility in vitro and might induce detrusor overactivity in vivo [15]. Furthermore, Wang et al showed that passive transfer of $\operatorname{IgG}$ with inhibitory antiM3R activity produced a paradoxical increase in contractile responses of detrusor strips to cholinergic stimulation. They also showed that features of cholinergic hyperresponsiveness were associated with increased postsynaptic M3R expression. They concluded that there was initial inhibition of parasympathetic neurotransmission by antagonistic autoantibodies to M3R, which produced a compensatory increase in M3R expression in vivo, causing enhanced cholinergic responses during bladder distention and resulting in detrusor overactivity. Immunofluorescence examination showed increased expression of M3R in the urinary bladder of the IgG-injected mice, which might play an important role in the pathogenesis of OAB [10].

It may be speculated that significantly higher rates of daytime frequency and nocturia observed in our pSS group might have resulted from excessive liquid intake in order to reduce mouth dryness. In line with this view, daily liquid intake in pSS group was higher than NHC group. However, daily liquid intake was not significantly different between pSS subgroups with and without OAB. So, significantly higher rates of daytime frequency and nocturia can not be explained only by means high liquid intake, and might be considered as the results of OAB.

Sacco et al reported a clinical case of unstable bladder in association with pSS and noted a moderate response to anticholinergic drugs [16]. Walker et al. assessed irritative and obstructive symptoms in patients with SS and osteoarthritis (OA) utilizing American Urology Symptom Index (AUA-7) scoring. The rate of patients with a score of 20 and above was $61 \%$ in patients with $\mathrm{SS}$, whereas it was found to be $40 \%$ in those with OA. They found out that irritating lower urinary tract symptoms (urinary frequency, urgency, bladder pain and nocturia) were significantly higher in patients with $\mathrm{SS}$ as compared to those with OA, while no significant dif- ference was determined in terms of obstructive symptoms [2].

Lee et al studied irritating bladder symptoms of $23 \mathrm{fe}-$ male patients with SS. They observed that $87 \%$ of the patients had frequency, 66\% nocturia and 52\% urgency. Defining polyuria as total daily urine output more than 3,000 $\mathrm{mL}$, they noted polyuria in four patients (17\%) based upon the voiding diary; average voided volume was $294 \mathrm{~mL}$ [17]. However we observed no polyuria in our patients. In our study; 24-hour average voided volume was $1,964 \mathrm{~mL}$ in patients with $\mathrm{SS}$, and $1,873 \mathrm{~mL}$ in the NHC group $(\mathrm{P}<0.05)$, while maximal urination volume was $189 \mathrm{~mL}$ in the pSS group, and $398 \mathrm{~mL}$ in the NHC group $(\mathrm{P}<0.05)$.

Haarala et al mailed a questionnaire to $46 \mathrm{pSS}$ patients regarding possible urinary complaints. Of the 36 responders, $14 \%$ reported severe and recurrent urinary symptoms, and this was significantly higher than the age and sex matched control population [18]. Another study compared rheumatoid arthritis patients having secondary SS with healthy controls using American Urological Association Symptom Index (AUASI). Both overall AUASI score and the rate of urinary frequency were significantly higher in secondary SS group compared to healthy controls $(\mathrm{P}<0.05)[3]$.

Despite all these previous studies claiming increased frequency of urinary symptoms and/or OAB in pSS, Sankar et al failed to confirm these findings. They compared pSS patients with xerostomic controls, reported that urinary symptoms might be secondary to increased fluid intake due to sicca symptoms. A total of 63 pSS patients and 38 controls were studied by a questionnaire plus urine analysis and salivary flow measurement. Despite a trend toward increased urinary symptoms among pSS patients, there was no overall significant difference between pSS subjects and xerostomia controls. Nocturia was correlated only with salivary flow among the xerostomia group, which suggested that factors other than xerostomia might be important to cause nocturia in pSS patients [19]. 
In conclusion, significantly higher OAB-V8 score, daytime frequency and nocturia were observed in pSS patients compared to the NHC group. Nocturia and frequent urination are important symptoms that disturb the comfort of daily life. We believe that all these findings implicate the high frequency of $\mathrm{OAB}$ in pSS. Increased liquid intake because of xerostomia alone cannot explain our findings. Since OAB is a treatable symptom complex, it should not be ignored, and while managing patients with pSS, possible symptoms of lower urinary system and $\mathrm{OAB}$ should be always be considered.

\section{Acknowledgement}

Financial disclosures: I certify that all conflicts of interest, including specific financial interests and relationships and affiliations relevant to the subject matter or materials discussed in the manuscript (eg, employment/affiliation, grants or funding, consultancies, honoraria, stock ownership or options, expert testimony, royalties, or patents filed, received, or pending), are the following: None.

\section{Funding/Support and Role of the Sponsor}

None.

\section{Disclosure Statement}

The authors declare that they have no conflict of interest.

\section{References}

1. Yu HJ, Lee WC, Lee KL, Chen MY, Chen CY, Chen J. Voiding dysfunction in women with systemic lupus erythematosus. Arthritis Rheum. 2004;50(1):166-172.

2. Walker J, Gordon T, Lester S, Downie-Doyle S, McEvoy $\mathrm{D}$, Pile $\mathrm{K}$, Waterman $\mathrm{S}$, et al. Increased severity of lower urinary tract symptoms and daytime somnolence in primary Sjogren's syndrome. J Rheumatol. 2003;30(11):2406-2412.

3. Lee KL, Chen MY, Yeh JH, Huang SW, Tai HC, Yu HJ. Lower urinary tract symptoms in female patients with rheumatoid arthritis. Scand J Rheumatol. 2006;35(2):96101.

4. Jonsson R, Haga H, Gordon TP. Sjögren's sydrome. In Koopman WJ, editor. Arthritıs and allied conditions. 14th ed. Philadelphia: Lippincott Williams\&Wilkins; 2001;1736-1759.

5. Milsom I, Stewart W, Thuroff J. The prevalence of overac- tive bladder. Am J Manag Care. 2000;6(11 Suppl):S565-573.

6. Stewart WF, Van Rooyen JB, Cundiff GW, Abrams P, Herzog AR, Corey R, Hunt TL, et al. Prevalence and burden of overactive bladder in the United States. World J Urol. 2003;20(6):327-336.

7. Cardozo L. Systematic review of overactive bladder therapy in females. Can Urol Assoc J. 2011;5(5 Suppl 2):S139-142.

8. Robinson D, Cardozo L. Overactive bladder: diagnosis and management. Maturitas. 2012;71(2):188-193.

9. Hocevar A, Tomsic M, Praprotnik S, Hojnik M, Kveder T, Rozman B. Parasympathetic nervous system dysfunction in primary Sjogren's syndrome. Ann Rheum Dis. 2003;62(8):702-704.

10. Wang F, Jackson MW, Maughan V, Cavill D, Smith AJ, Waterman SA, Gordon TP. Passive transfer of Sjogren's syndrome IgG produces the pathophysiology of overactive bladder. Arthritis Rheum. 2004;50(11):3637-3645.

11. Coyne K, Revicki D, Hunt T, Corey R, Stewart W, Bentkover J, Kurth H, et al. Psychometric validation of an overactive bladder symptom and health-related quality of life questionnaire: the OAB-q. Qual Life Res. 2002;11(6):563-574.

12. Coyne KS, Zyczynski T, Margolis MK, Elinoff V, Roberts RG. Validation of an overactive bladder awareness tool for use in primary care settings. Adv Ther. 2005;22(4):381-394.

13. Vitali C, Bombardieri S, Jonsson R, Moutsopoulos HM, Alexander EL, Carsons SE, Daniels TE, et al. Classification criteria for Sjogren's syndrome: a revised version of the European criteria proposed by the American-European Consensus Group. Ann Rheum Dis. 2002;61(6):554558.

14. Ouslander JG. Management of overactive bladder. N Engl J Med. 2004;350(8):786-799.

15. Waterman SA, Gordon TP, Rischmueller M. Inhibitory effects of muscarinic receptor autoantibodies on parasympathetic neurotransmission in Sjogren's syndrome. Arthritis Rheum. 2000;43(7):1647-1654.

16. Sacco F, Rigon G, Sacchini D. [Unstable bladder and Sjogren syndrome. Clinical case]. Minerva Med. 1996;87(5):257-259.

17. Lee KL, Dong CS, Chen MY, Ho CH, Tai HC, Hung SF, Yu HJ. Multifactorial causes of irritating bladder symptoms in patients with Sjogren's syndrome. Neurourol Urodyn. 2011;30(1):97-101.

18. Haarala M, Alanen A, Hietarinta M, Kiilholma P. Lower urinary tract symptoms in patients with Sjogren's syndrome and systemic lupus erythematosus. Int Urogynecol J Pelvic Floor Dysfunct. 2000;11(2):84-86.

19. Sankar V, Kok M, Leakan RA, Pillemer SR. The Prevelance of ürinary tract symtoms in patients with Sjogren's syndrome. Arthritis Rheum. 2001;44:142. 